\title{
Burden of acute toxicities in head-and-neck radiation therapy: A single-institutional experience
}

\author{
Sandeep Muzumder, Nirmala Srikantia,Avinash H. Udayashankar, Prashanth Bhat Kainthaje, M. G. John Sebastian
}

\begin{abstract}
Introduction: Combined modality therapy is the standard of care in locally advanced head-and-neck cancer (HNC). The incidence of acute toxicities increases with additional therapy. The present study investigated the incidence and patterns of mucositis, dysphagia, aspiration, feeding tube use, admission for supportive care, and treatment compliance in patients with HNC treated curatively with radiation therapy (RT) with or without chemotherapy.Methods and Material: A retrospective review of 164 consecutive HNC patients treated with RT at St. John's Medical College Hospital, Bengaluru, from January 20 I 3 to June 2017 was done. Results: A total of I 48 HNC patients were treated with a curative intent and I22 (82.4\%) were locally advanced HNC. Combined Modality treatment was received by $119(80.4 \%)$ patients. Eighty-four $(56.7 \%)$ patients were treated by concurrent chemo-radiation. IMRT technique was used in 125 (84.5\%) patients. The incidence of grade 3-4 mucositis, dysphagia and aspiration was $25 \%$, $46 \%$, and $10 \%$, respectively. Nasogastric tube feeding was necessitated in $18.9 \%(n=28)$ and $27 \%(n=40)$ required inpatient admission for supportive care.Twenty-nine $(19.6 \%)$ patients did not complete planned RT dose and 46 (3I\%) patients had unscheduled RT break (>2days). Fifty-six (66.7\%) patients did not receive planned chemotherapy. Conclusions: Acute toxicity due to RT in HNC remains a challenge despite using modern techniques. A significant proportion of patients require supportive therapy for more than 12 weeks and did not complete the scheduled treatment.
\end{abstract}

Key words:Acute toxicity, dysphagia, head-and-neck cancer, mucositis, radiation therapy

\section{Introduction}

Head-and-neck cancer (HNC) is the most common malignancy in India, and $83 \%$ of patients present with Stage III and IV HNC. ${ }^{[1]}$ Radiation therapy (RT) is the most commonly used and effective cancer-directed therapy for advanced HNC. In modern oncology practice, combined modality therapy (CMT) is the mainstay of cancer management. Concurrent chemoradiation (CCRT) is the standard of care, providing an absolute benefit of $6.5 \%$ compared with RT alone ${ }^{[2]}$ However, the incidence of acute toxicities, including mucositis and dysphagia, increased two folds in patients who underwent CCRT compared with those who underwent RT alone. ${ }^{[3,4]}$ In the European Organization for Research and Treatment of Cancer 22931 trial, the proportion of severe adverse events (Grade $\geq 3$ ) was $41 \%$ in the CCRT group versus $21 \%$ in the RT-only group. ${ }^{[3]}$ The assessment of adverse events is subjective and varies between trials, even when a similar treatment protocol is used. In the Radiation Therapy Oncology Group 9501 trial, the incidence of severe acute adverse events was $77 \%$ in the CCRT group versus $34 \%$ in the RT-only group. ${ }^{[4]}$ The incidence of aspiration was also reported to be high after head and neck radiation in India because patients present with advanced stages at diagnosis. ${ }^{[5]}$ Because aspiration is related to dysphagia and mucositis, the incidence of aspiration is also likely to increase with CMT. With advances in RT techniques, intensity-modulated RT (IMRT) is now widely used for managing HNC. Although IMRT has reduced the long-term incidence of xerostomia, the same cannot be indicated for acute toxicities such as mucositis and dysphagia. ${ }^{[6,7]}$

The present study investigated the incidence and patterns of mucositis, dysphagia, aspiration, feeding tube use, admission for supportive care, and treatment compliance in patients with HNC treated curatively with RT with or without chemotherapy.

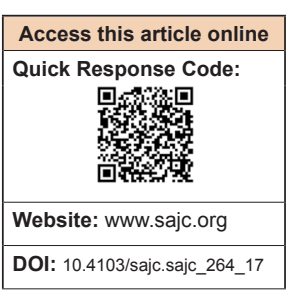

\section{Patients and Methods}

\section{Study design and setting}

This retrospective study was conducted at the Department of Radiation Oncology at St. John's Medical College and Hospital, Bengaluru, India, after obtaining approval from the institutional ethical committee. All patients with HNC treated between January 2013 and June 2017 were recruited into this study. All patients who received palliative RT and re-irradiation were excluded from the study. All patient management decisions were discussed among the members of the multidisciplinary tumor board. Patients received RT and chemotherapy according to standard guidelines and practices. All patients underwent a thorough clinical and endoscopic evaluation. A biopsy or fine-needle aspiration cytology and a computed tomography (CT) scan were performed before starting the treatment. Pretreatment baseline tests for complete blood count, renal function, liver function, and creatinine clearance were performed. Cancer staging was performed according to the guidelines of the American Joint Committee on Cancer, Seventh Edition (2010). Data were collected by mining radiotherapy review charts and follow-up records.

\section{Treatment}

Patients were immobilized using a thermoplastic mask; subsequently, a CT scan with or without an intravenous (IV) iodinated contrast was performed for all patients. IMRT or three-dimensional conformal RT (3D-CRT) with 6MV photons was used to deliver RT. No patient was treated using a conventional simulator or two-dimensional planning. In the adjuvant setting, RT was started within 6 weeks of surgery following adequate wound healing. Total doses of 66-70 and 54-66 Gy were administered in radical and adjuvant settings,

This is an open access journal, and articles are distributed under the terms of the Creative Commons Attribution-NonCommercial-ShareAlike 4.0 License, which allows others to remix, tweak, and build upon the work non-commercially, as long as appropriate credit is given and the new creations are licensed under the identical terms.

For reprints contact: reprints@medknow.com

How to cite this article: Muzumder S, Srikantia N, Udayashankar $\mathrm{AH}$ Kainthaje PB, John Sebastian MG. Burden of acute toxicities in head-and-neck radiation therapy: A single-institutional experience. South Asian J Cancer 2019;8: 120-3.
Department of Radiation Oncology, St. John's Medica College and Hospital, Bengaluru, Karnataka, India Correspondence to: Dr. Sandeep Muzumder, E-mail: sandeepmuzumder@gmail.com 
respectively. RT was administered 5 days a week. The CCRT schedule was either weekly cisplatin or 3-weekly cisplatin based on the preference of the treating oncologist. Cisplatin was administered at a flat dose of $50 \mathrm{mg}$ in the weekly schedule and $100 \mathrm{mg} / \mathrm{m}^{2}$ in the 3-weekly schedule. None of the patients received chemotherapy after RT completion.

Follow-up

All patients were reviewed at least twice a week while undergoing RT (every Monday and Thursday). After completion of the scheduled treatment, patients were followed up weekly until acute reactions subsided and then monthly until 6 months. At each follow-up, the grading of toxicities was recorded in the RT chart.

\section{Study outcome}

The primary objective of this study was to determine the incidence and patterns of mucositis, dysphagia, aspiration, feeding tube use, and inpatient (IP) admission for supportive care. Toxicity grading was performed using Common Terminology Criteria for Adverse Events 4.03. Mucositis requiring morphine intake for pain control, IV hydration, nasogastric (NG) tube insertion for poor oral intake, IP admission, or IV antibiotic use was labeled as Grade 3. Dysphagia requiring NG tube insertion, IV hydration, morphine intake, or IP admission for the management of associated aspiration was labeled as Grade 3. A patient with Grade 3 pain was defined as any patient requiring morphine for treatment-related pain. The maximum grade of aspiration, dermatitis, nausea, vomiting, anorexia, weight loss, and pain was recorded at each follow-up. To assess compliance, the median radiation dose received and the percentage of patients receiving planned radiation or chemotherapy were calculated.

\section{Statistical analysis}

The study population was selected using convenience sampling. Data were analyzed using Microsoft Excel 2016. All categorical data were presented as frequencies and percentages. All continuous data were presented as median and range based on distribution. To depict the patterns of mucositis and dysphagia, an exact number of patients having Grade 1,2 , or $\geq 3$ toxicity in each evaluated week was entered, and a figure was derived in Microsoft Excel.

\section{Results}

From 164 patients with HNC who underwent RT, we excluded 14 patients who received palliative RT, 1 patient who underwent emergency tracheostomy due to disease progression after the second fraction of RT and treatment refusal, and 1 patient who had recurrent pleomorphic adenoma of the parotid gland. Finally, the remaining 148 patients were analyzed. The baseline characteristics of the 148 patients are listed in Table 1. Of the 148 patients, 125 (84.5\%) received IMRT, whereas the remaining patients received 3D-CRT. Furthermore, 119 (82\%) patients received CMT and $85(57.4 \%)$ patients received chemotherapy. One patient received neoadjuvant chemotherapy (NACT), followed by RT alone, and 2 patients received NACT, followed by CCRT. A total of 84 patients who underwent CCRT received cisplatin.

The incidence of acute toxicities is listed in Table 2. The following acute adverse events of $\geq$ Grade 3 occurred in the study population (with the incidence provided parenthetically):
Table 1: Baseline characteristics

\begin{tabular}{|c|c|c|}
\hline Characteristics & & $n(\%)$ \\
\hline \multicolumn{3}{|l|}{ Age (years) } \\
\hline Median (range) & & $58(17-85)$ \\
\hline \multicolumn{3}{|l|}{ Sex } \\
\hline Male & & $107(72.3)$ \\
\hline Female & & $48(27.7)$ \\
\hline \multicolumn{3}{|l|}{ Site } \\
\hline Oral cavity & & $47(31.8)$ \\
\hline Oropharynx & & $22(14.9)$ \\
\hline Hypopharynx & & $20(13.5)$ \\
\hline Larynx & & $33(22.3)$ \\
\hline Salivary gland & & $10(6.7)$ \\
\hline Other sites & & $16(10.8)$ \\
\hline \multicolumn{3}{|l|}{ Stage grouping } \\
\hline I-II & & $26(17.6)$ \\
\hline III-IVB & & $122(82.4)$ \\
\hline \multicolumn{3}{|l|}{ Tobacco/alcohol use } \\
\hline Tobacco chewing & & $51(34.5)$ \\
\hline Smoking & & $65(43.9)$ \\
\hline Alcohol & & $25(16.9)$ \\
\hline \multicolumn{3}{|l|}{ Comorbidities } \\
\hline Diabetes mellitus & & $33(22 / 3)$ \\
\hline $\mathrm{CCI}$, median (range) & & $4(2-9)$ \\
\hline \multicolumn{3}{|l|}{ Treatment modality } \\
\hline RT alone & & $29(19.6)$ \\
\hline PORT & & $37(25.0)$ \\
\hline $\mathrm{RT}+\mathrm{CT}$ & & $64(43.2)$ \\
\hline $\mathrm{PORT}+\mathrm{CT}$ & & $18(12.2)$ \\
\hline \multicolumn{3}{|c|}{$\begin{array}{l}\text { Data are presented as } n(\%) \text { unless specified otherwise. Other sites included } \\
\text { CUP }(n=4) \text {, sinonasal }(n=4) \text {, ear }(n=3) \text {, nasopharynx }(n=3) \text {, and thyroid }(n=2) \text {. } \\
\text { Stage grouping (AJCC, Seventh Edition). CUP=Cervical node with unknown } \\
\text { primary, CCI-Charlson Comorbidity Index; PORT=Postoperative radiation therapy; } \\
\text { CT=Chemotherapy, RT=Radiation therapy }\end{array}$} \\
\hline \multicolumn{3}{|c|}{ Table 2: Incidence of acute toxicities } \\
\hline Characteristics & Any grade $(\%)$ & Grade $\geq 3(\%)$ \\
\hline Mucositis & $114(89.1)$ & $33(25.8)$ \\
\hline Dysphagia & $135(98.5)$ & $59(46.1)$ \\
\hline Aspiration & $81(54.7)$ & $14(9.5)$ \\
\hline Dermatitis & $138(93.2)$ & $8(5.4)$ \\
\hline Nausea & $47(31.8)$ & 0 \\
\hline Vomiting & $71(47.9)$ & 0 \\
\hline Anorexia & $74(50.0)$ & 0 \\
\hline Weight loss & $129(87.2)$ & $5(3.4)$ \\
\hline Pain & $132(89.2)$ & $22(14.8)$ \\
\hline
\end{tabular}

mucositis $(n=33,25.8 \%)$, dysphagia $(n=59,46.1 \%)$, aspiration $(n=14,9.5 \%)$, dermatitis $(n=8,5.4 \%)$, pain $(n=22,14.8 \%)$, and weight loss $(n=5,3.4 \%)$. The median weight loss was $4 \mathrm{~kg}$ (range: 1-15). Grade 3 dermatitis most commonly occurred at the tracheostomy or tracheostomal site. No patient developed Grade 3 nausea, vomiting, or anorexia. Symptomatic mucositis (Grade $\geq 2$ ) started from week 2 , peaked at week 3 , persisted for 6 weeks, declined by week 10 , and subsided by week 16 . Half of the patients required management of symptomatic mucositis (Grade $\geq 2$ ) for 10 weeks [Figure 1]. Two patients developed Grade IV mucositis. Both the patients had carcinoma of the oral cavity and received CCRT. The pattern of dysphagia was more severe than that of mucositis, with $90 \%$ patients requiring symptomatic treatment for $>7$ weeks [Figure 2]. At 24 weeks after therapy initiation, three patients had persistent Grade III dysphagia. 


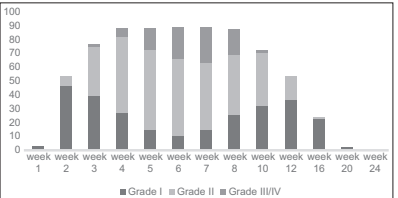

Figure 1: Pattern of mucositis over time

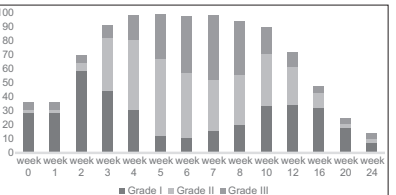

Figure 2: Pattern of dysphagia over time
In $28(18.9 \%)$ patients, an NG tube was inserted during RT due to dysphagia. Furthermore, $23(15.5 \%)$ and $40(27 \%)$ patients required IV hydration on a day-care basis and IP admission for supportive care, respectively. The median duration of admission was 6 (range: 2-25) days.

All the patients, except one, received conventional fractionation. One patient received hyper-fractionated RT for acinic cell carcinoma of the parotid gland. One-fifth of the patients $(n=29$, $19.6 \%$ ) did not receive the planned RT dose. Among 29 patients, toxicity $(n=19)$ was the major cause of incomplete treatment, followed by treatment refusal $(n=9)$ and disease progression $(n=1)$. An unscheduled treatment break ( $>2$ days) during RT occurred in $46(31 \%)$ of the 148 patients. Most of the patients $(n=41)$ required a break due to toxicity and 5 patients opted for a break due to social reasons. The median duration of RT was 44 days (range: 1-66). A total of $56(66.7 \%)$ patients did not receive planned chemotherapy.

\section{Discussion}

In the present study, we found that the majority (82.4\%) of the patients with HNC treated with curative intent had Stage III-IVB disease. Furthermore, $80.4 \%$ and $56.7 \%$ of the patients received CMT and CCRT, respectively. Of all patients, 50\% developed mucositis and dysphagia requiring symptomatic management for 12 weeks starting from the $3^{\text {rd }}$ week of RT. Therefore, many patients required symptomatic treatment for 6 weeks after completing RT. One-fourth of the patients (40 of the 148 patients) required IP admission for supportive care.

The retrospective design of this study is a major limitation. The grading of acute toxicities, such as aspiration, was subjective and depended on the treating oncologist. RT alone was administered to 29 patients, of whom 16 had early glottic cancer with a small RT field and 7 were unfit for chemotherapy due to the presence of a comorbidity. Therefore, the comparison of the RT alone group with the CMT group was not feasible.

Despite advancements in the RT technique, acute toxicity continues to be a major challenge in head-and-neck RT. In the present study, $84.5 \%$ of the patients received IMRT. The burden of acute toxicity is expected to remain high, as an increasing number of patients receive CMT. In a review conducted by Trotti et al., the incidence of severe mucositis was $34 \%$ in patients who underwent conventional RT and the incidence increased in patients who underwent aggressive treatment modalities ${ }^{[8]}$ The rate of hospitalization due to mucositis was $16 \%$ in the overall study population and $32 \%$ in patients with altered fractionation. The RT regimen of $11 \%$ of the patients was interrupted or modified due to the development of mucositis. The present study population had higher rates of hospitalization and treatment interruptions.

Moroney et al. reported the occurrence of severe dysphagia in $29 \%$ of patients treated with IMRT and concurrent chemotherapy. ${ }^{[9]}$ At 12 weeks after RT completion, posttreatment complete blood count, renal function, liver function, and creatinine clearance returned to pretreatment baseline levels in the patients who required supportive care for symptomatic toxicity (Grade $\geq 2$ ). The addition of chemotherapy doubled the rates of most acute toxicities. The results of this study highlight the need for supportive care due to acute toxicities despite using IMRT.

With half and one-fourth of the patients requiring supportive care and IP admission for severe toxicity, respectively, the burden on hospitals treating patients with $\mathrm{HNC}$ is substantial. This might have a major implication because HNC constitutes $21.2 \%$ of cancers in India. ${ }^{[10]}$ We can only speculate the direct and indirect costs involved due to acute toxicities. An Indian study conducted in a public sector hospital reported that treatment costs doubled when using combined RT and chemotherapy compared with when using RT alone. ${ }^{[1]]}$ Majority of patients $(76 \%)$ encounter financial problems while undergoing cancer treatment in India. ${ }^{[12]}$ Future prospective studies are required to determine the burden of treatment-related acute toxicities in patients with $\mathrm{HNC}$ in India.

\section{Conclusion}

Acute toxicity remains a challenge in RT for HNC despite the use of contemporary RT techniques. Approximately 50\% of the patients developed mucositis and dysphagia requiring symptomatic management for 12 weeks starting from the $3^{\text {rd }}$ week of RT initiation. A high proportion of patients required supportive therapy and did not complete the scheduled treatment.

\section{Acknowledgment}

We acknowledge and thank all staff at the Department of Radiation Oncology, Surgical Oncology, Medical Oncology, ENT, and Pain and Palliative Care at St John's Medical College and Hospital, Bengaluru, India.

\section{Financial support and sponsorship}

This study was financially supported by the Department of Radiation Oncology, St John's Medical College and Hospital, Bengaluru, India.

\section{Conflicts of interest}

There are no conflicts of interest.

\section{References}

1. Kaur J, Mohanti BK, Muzumder S. Clinical audit in radiation oncology: Results from one academic centre in Delhi, India. Asian Pac J Cancer Prev 2013; 14:2829-34.

2. Pignon JP, le Maître A, Maillard E, Bourhis J, MACH-NC Collaborative Group. Meta-analysis of chemotherapy in head and neck cancer (MACH-NC): An update on 93 randomised trials and 17,346 patients. Radiother Oncol 2009;92:4-14.

3. Bernier J, Domenge C, Ozsahin M, Matuszewska K, Lefèbvre JL, Greiner RH, et al. Postoperative irradiation with or without concomitant chemotherapy for locally advanced head and neck cancer. N Engl J Med 2004;350: 1945-52.

4. Cooper JS, Pajak TF, Forastiere AA, Jacobs J, Campbell BH, Saxman SB, et al. Postoperative concurrent radiotherapy and chemotherapy for high-risk squamous-cell carcinoma of the head and neck. N Engl J Med 2004;350: 1937-44.

5. Madan R, Kairo AK, Sharma A, Roy S, Singh S, Singh L, et al. Aspiration pneumonia related deaths in head and neck cancer patients: A retrospective analysis of risk factors from a tertiary care centre in North India. J Laryngol Otol 2015;129:710-4.

6. Gupta T, Agarwal J, Jain S, Phurailatpam R, Kannan S, Ghosh-Laskar S, et al. Three-dimensional conformal radiotherapy (3D-CRT) versus South Asian Journal of Cancer $\bullet$ Volume 8 Issue 2 April-June 2019 
intensity modulated radiation therapy (IMRT) in squamous cell carcinoma of the head and neck: A randomized controlled trial. Radiother Oncol 2012; 104:343-8.

7. Marta GN, Silva V, de Andrade Carvalho H, de Arruda FF, Hanna SA, Gadia R, et al. Intensity-modulated radiation therapy for head and neck cancer: Systematic review and meta-analysis. Radiother Oncol 2014;110:9-15.

8. Trotti A, Bellm LA, Epstein JB, Frame D, Fuchs HJ, Gwede CK, et al. Mucositis incidence, severity and associated outcomes in patients with head and neck cancer receiving radiotherapy with or without chemotherapy: A systematic literature review. Radiother Oncol 2003;66:253-62.

9. Moroney LB, Helios J, Ward EC, Crombie J, Wockner LF, Burns CL, et al.
Patterns of dysphagia and acute toxicities in patients with head and neck cancer undergoing helical IMRT \pm concurrent chemotherapy. Oral Oncol 2017;64:1-8.

10. Alam MS, Siddiqui SA, Perween R. Epidemiological profile of head and neck cancer patients in Western Uttar Pradesh and analysis of distributions of risk factors in relation to site of tumor. J Cancer Res Ther 2017; 13:430-5.

11. Mohanti BK, Mukhopadhyay A, Das S, Sharma K, Dash S. The economic burden of cancer. Econ Polit Wkly 2011;43:112-8.

12. Nair KS, Raj S, Tiwari VK, Piang LK. Cost of treatment for cancer: Experiences of patients in public hospitals in India. Asian Pac J Cancer Prev 2013; 14:5049-54. 